\title{
Dynamic Compression in Affective Haptics
}

\author{
ESTHER FOO, Doctoral Student, Human Factors and Ergonomics, University of Minnesota
}

BRAD HOLSCHUH, Doctoral Advisor, University of Minnesota

\author{
CCS Concepts: • Human Computer Interaction (HCI) $\rightarrow$ Interaction Paradigms \\ KEYWORDS: Wearable Technology, Affective Haptics, Haptic Communication, Human Factors
}

\section{INTRODUCTION}

The sense of touch, through stimulation of the skin by mechanical, thermal, chemical or electrical stimuli, is an important way to perceive and represent reality. A variety of sensations can be elicited, such as temperature, vibration, pressure, tapping, and pain. One of these sensations is counter-pressure, or compression. Compression is a ubiquitous sensation; from feeling pressure on the soles of our feet, to wearing form-fitted clothing, or receiving a hug. It is also used in a variety of applications related to human health, such as graduated stockings to improve circulation and anti-gravity suits worn by astronauts subjected to high acceleration forces. Further, interventions such as weighted vests, massage therapies, and infant swaddling, all benefit from the use of compression to positively affect the wellbeing and emotion of users. Such applications have seen the benefits of strategically-applied compression by decreasing anxiety, promoting feelings of calmness and improving alertness [1]-[4]. Despite touch being the first sensory system to develop in all animal species and the skin being the largest sensory organ, the experience of touch, including compression, is vastly understudied [4]. Recent work in this area have shown the development of a new breed of garment-based dynamic compression technology, capable of producing computermediated compression effects on the body, an advancement that makes it uniquely possible to drive this domain of study forward [5]. Therefore, this position paper outlines an ongoing PhD project, seeking to investigate the use of computer-mediated on-body compression systems to expand our understanding of the objective and subjective effects of compression on a user.

\section{PROBLEM DOMAIN(S) AND RELATED WORK}

Haptics are studied in many different domains, perhaps most significantly as a communication modality. Given the proliferation of electronic technology, the visual and auditory systems have been shouldering significant attentional demands for conscious interactions. As such, researchers are turning to haptics as a secondary form of communication. Haptic communication reconstructs the sense of touch by inducing forces on a user which may include taps, vibration, point pressure and distributed pressure. Vibrotactile displays are most commonly used but they require high attentional demands and may be disruptive. Several studies have explored pressure feedback using point pressure actuators but such localized haptic displays only cover a small area, which may be limiting in its communication abilities [6]. Distributed pressure, such as compression, is advantagous by being less attention-demanding [6] (suited for ambient feedback), able to invoke a wide range of attention capture (subtle to forceful) [7], and is similar to human behaviors (e.g., a hug). Some work in this area include Baumann et. al.'s Servosqueeze using tensioning of a passive wrist

Permission to make digital or hard copies of all or part of this work for personal or classroom use is granted without fee provided that copies are not made or distributed for profit or commercial advantage and that copies bear this notice and the full citation on the first page. Copyrights for components of this work owned by others than ACM must be honored. Abstracting with credit is permitted. To copy otherwise, or republish, to post on servers or to redistribute to lists, requires prior specific permission and/or a fee. Request permissions from Permissions@acm.org.

UbiComp/ISWC'18 Adjunct, October 8-12, 2018, Singapore, Singapore

(C) 2018 Association for Computing Machinery.

ACM ISBN 978-1-4503-5966-5/18/10...\$15.00

https://doi.org/10.1145/3267305.3267312 
strap to gain a users's attention [8], Chinello et. al.'s HapBand with moving plates for remote communication [9], and Pohl et. al.'s Squeezeback inflatable wrist cuff that uses compression for notifications [7].

A plethora of sensations can be evoked by touch, but perhaps more importantly, research has shown that touch is able to communicate distinct emotions using different tactile behaviors, which demonstrates the richness of the physical properties of touch [10]. Further, for many years, the field of occupational therapy (OT) has employed strategies such as holding, swaddling, hugging, and squeezing (all of which are related to compression), to provide physical and emotional benefit (e.g., by encouraging stress reduction, relief of muscle tension, reduction of mental anxiety, and an overall improved feeling of well-being) [4]. It is used as an intervention with clinical populations experiencing autism spectrum disorder (ASD) [3], [11]. These examples demonstrate a plausible link between haptic stimulation and emotion, which is developing into this new field of affective haptics, an area that focuses on the 'design of systems that can elicit, influence, or enhance the emotional state of a human through the sense of touch' [12]. There are several forms of approaches in regards to research in affective haptics. The first uses touch as a form of affective haptic expression, typically involving a participant communicating an emotion through a haptic device, while another identifies the encoded emotion given the haptic affordances [13]. In contrast, the second group of studies are related to social mediated touch, with a focus on enhancing communication channels in remote communication contexts. Mueller et. al.'s 'Hug Over a Distance' inflatable vest allows an intimate couple separated by distance to communicate emotional messages through on-body compression [14], Teh et. al.'s 'Huggy Pajama' facilitates remote communication between a parent and a child [15], Tsetserukou et. al.'s 'iFeel_IM!' system consists of various haptic devices that reproduces the feelings of social touch experienced in a virtual environment by means of haptic stimulation in the real world [12], and Wang and Quek's haptic pressure sleeve was used to enhance emotionally-intense moments in a storytelling scenario [16].

Sitting within the intersection of the fields of haptic communication and affective haptics, lies another form of approach (one that this paper takes), which uses haptics to influence one's emotions. This approach largely intersects with persuasive computing, a field that broadly describes technology that is aimed at changing the attitudes and/or behaviors of users through persuasion and social influence, without the use of coercion or deception [17]. Currently, most systems convey information explicitly to the user and require users to consciously make a decision to act upon the provided information (e.g. fitness trackers). This is a form of persuasive technology, but the persuasion is done indirectly since it still requires conscious effort on the user's part for a change to occur. Conversely, a more direct form of persuasion would be to induce changes without conscious action from the user. As mentioned, compression has been demonstrated as an effective intervention for those with ASD, demonstrating the potential for a direct link between stimulation (e.g. compression) and attitude/ behavioral changes (e.g. calming effect) [11]. If coupled with a feedback mechanism that can autonomously detect context or the onset of triggers, such actuatable compression systems have huge potential for growth as a novel form of persuasive computing.

\section{ORIGINAL KEY IDEA AND THESIS RESEARCH QUESTIONS}

\subsection{Gaps in current research landscape}

Studies using compression as a haptic modality and its affective abilities intersects many application areas including human-computer interaction, affective haptics, wearable technology, and OT. However, to truly maximize the use of compression in such a wide variety of applications, the effects of compression on the human body, especially when used as an interaction modality, must be better understood. Broadly, the experience of compression can be modulated with varying inputs including application location, intensity, rate, and duration. However, there is a lack of rigorous study with respective to proper stimulation for a desired experience. Furthermore, in a haptic communication context, there have been several studies using compression as a form of feedback but they are largely limited to wrist-based technologies [8], [9], and there is a lack of focus on using a garment form factor to distribute compressive forces onto larger body 
areas (e.g., torso). Garments are ideal for creating compression due to the large direct-acting surface area, close proximity to the body, as well as the social ubiquity of clothing that provides a physically intimate yet low-profile solution compared to conventional wearable gadgets [18]. Current garment-based compression systems are typically either (1) passive, non-adjustable garments exerting compression through added weights or stretch (e.g. weighted vests, elastic clothing), or (2) dynamic garments constricting the user [15]. The two most common methods of actuation for dynamic garments include the tensioning of straps through servos, or pneumatic inflatables. However, both designs suffer from functional or usability issues, such as limitations in donning/ doffing, mobility, portability, bulk, and noise (by air pumps for inflatables) [19]. Further, one of the main deterrents that prevents rigorous study of these problems is the lack of ability of current garment solutions to precisely/dynamically control the required compression generated.

\subsection{Key Idea's Supporting Evidence}

A potential solution to close this research gap is to develop computer-mediated compression garments that are dynamic, low-profile, and remotely-controllable. Specifically, the key idea is to create an innovative garment platform that abandons traditional wearable actuation schemes (e.g. servos, hydraulics, pneumatics) and instead shifts toward the use of embedded active materials (i.e., artificial muscles) that feature the ability to repeatably change shape when actuated. Recent research in active compression garment design has focused on form-fitting garments with integrated shape memory alloys (SMAs). SMA wires formed into spring-like actuators (that constrict when heated) have been shown to produce controllable compression (up to $225 \mathrm{mmHg}$ ) that scales with applied current [19]. SMA coil actuators are a particularly appealing solution in this context for a variety of reasons: first, their large active displacements can be used as a mechanism of donning and doffing loose garments while retaining the ability to dynamically apply compression; further, prior work has shown their active forces can be used to augment compression produced by passive garments [5]; finally, they can be easily and unobtrusively integrated into typical textile structures. Using this mechanism of computer-mediated compression, the objective and subjective effects of on-body dynamic compression can be rigorously studied.

\subsection{Research questions}

RQ1: Is it feasible to produce a computer-mediated active compression garment system that is simultaneously functional, remotely-controllable, untethered, and inconspicuous?

RQ2: How do the objective and subjective effects of on-body compression change with varying parameters (e.g. spatial distribution, magnitude, duration, rate of compression)?

RQ3: What implications do the objective/ subjective effects have on the prospect of computer-mediated compression as a haptic feedback mechanism?

RQ4: For which applications/contexts do computer-mediated compression make sense and how might the use of active compression garments affect traditional compression treatment paradigms?

\section{METHODOLOGICAL APPROACH CHOSEN}

Even though the technology to create dynamic compression now exists, the grounded design principles for upper body compression garments have yet to be clarified. Therefore, this work takes the broad approach of first understanding how users experience compression on various areas on the upper body: starting with a simple adjustable passive garment, followed by a technology-integrated active garment. Once those user experiences are better understood, the system (with user feedback incorporated) will be deployed to judge its effectiveness at modulating user affect in a scenario, on a healthy adult (ages 18-34) target population.

\subsection{Research roadmap}

To evaluate the feasibility of producing a computer-mediated active compression garment system that is simultaneously functional, remotely-controllable, untethered, and inconspicuous, two pilot studies with a 
passive and active garment prototype will be conducted, with a primary emphasis on feasibility of providing compression on different body locations (e.g. torso, shoulders, arms). First, an adjustable passive compression garment prototype will be created using readily available materials (e.g. hook and loop fasteners and passive straps to apply adjustable compression). Second, a dynamic compression garment incorporating SMA-based active materials as a compression actuation mechanism will be developed (RQ1, Phase 1). Both the passive and active garments will be used in small-scale user studies that qualitatively evaluate subjective experiences of users when exposed to variable upper-body compression.

Phase 2 of the investigation addresses two parallel research questions. The first tackles the variables important to understanding the design principles for upper body compression garments. As such, the experience of compression will be modulated through the delivery of variable compression location, rate, duration, and intensity profiles using the SMA-based garment architecture (RQ2). The effects of these varying inputs will be measured objectively (through biometric responses) and subjectively (through experiential user responses). The objective effects include measureable physiological data such as heart rate variability (HRV) and electrodermal activity (EDA) to determine if physiological responses are detectable, while the subjective effects will be collected through comfort and emotional user-reported responses. Further, spatial distribution data will also be captured to inform compression profiles on various users. The parallel research question is driven by the fact that the SMA-based garment used is inherently controllable, so there is an extra element of interest to the investigation: i.e., how might this form of compression stimulation be implemented in partnership with either an automated (computerized) or remote control (human) operator (RQ3). Given that, the SMA-based garment architecture will be developed with on-board power systems and wireless control via WiFi/Bluetooth compatible relays to allow computer-mediated compression inputs. The empirical knowledge gained from these research questions will be used to fine-tune the operating paradigms in dynamic computer-mediated compression.

Given the findings of the aforementioned variables (i.e. location, magnitude, duration, and rate of compression), a larger scale context-specific user experience study of computer-mediated compression will be performed (RQ4, Phase 3). Since evidence in the literature has pointed to the ability of compression to induce calming effects, a context-based experiment could be one that employs an arousal-inducing scenario. An example of this is the Stroop color word test, well-established sympathetic response-inducing method [20]. The idea here is to investigate if compression can be used to 'calm' a user, an application that has many implications in the field of OT. As before, both objective and subjective measures will be collected. The success of this study will be evaluated based on statistical differences in physiological and psychological data between groups that are wearing the garment or otherwise (control); we hypothesize that dynamic compression will provide reduced HRV and EDA compared to the control group. The study will be further divided into: (1) stationary, whereby the participant is seated with minimal movement and (2) mobile, whereby the participant is performing an activity that requires movement (e.g. walking). This is to ensure that the applied pressure is still perceivable (with intended effects maintained) when subjects are in motion, which is important in simulating an actual use scenario of a commercial garment.

\subsection{Research carried out so far}

Currently, the research in Phase 1 has been completed. In the first study $(n=5)$, compression was applied with a passive garment using hook-and-loop straps, strategically anchored throughout the garment and were free to move in various orientations (Figure 1). The subjective experiences of compression on the trunk of body and shoulder areas were differentiated (through different shoulder strap orientations). The study used a mixed-method approach with surveys, subjective interviews, and an informant design exercise. The results from this passive garment study yielded important design insights. In general, the garment was fairly well received, with valence measures trending towards positive and arousal values towards calm. In terms of shoulder strap orientation preference, the participants preferred either the 
having both straps cross their chest or straight down like backpack straps. Interestingly, these two compression distributions were vastly different, showing there may be larger individual differences in terms of perceived comfort than initially expected. One of the major themes gathered was the role of movement and breathing while compression is applied, i.e., the balance between compliance and stiffness of fabric. Given that the ultimate goal is to create a garment with integrated active SMA actuators, these variables are of important consideration. A compliant fabric would provide more comfort but reduces actuator efficiency; stiffer fabrics would be more stable and larger compression, but an overly stiff fabric may require precise actuator control to create desired pressures. Generally, users wanted more compression on the lower spine and sides and more flexibility in the front and shoulders for breathing and movement. The comments obtained were used to drive the design process of the dynamic SMA garment.

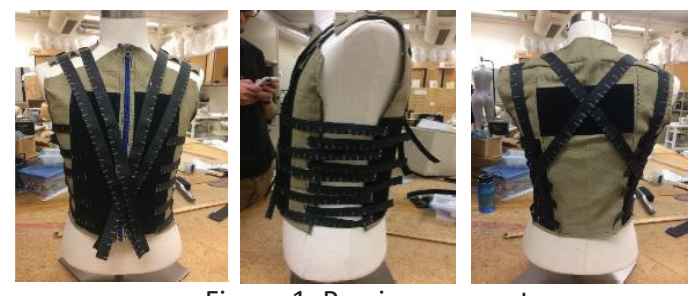

Figure 1: Passive garment

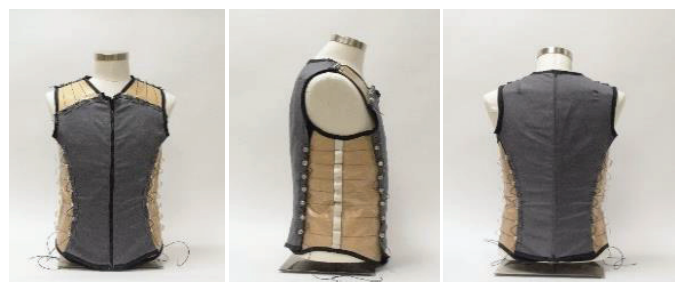

Figure 2: Active SMA garment

The second study aimed to understand how people respond to dynamic compression on the upper body, using a novel active compression garment system with embedded SMA actuators to produce controllable counter-pressures in the order of seconds (Figure 2). Following feedback from the passive garment study, the active garment design included more compliant fabrics, extended garment length, actuators placed away from garment edges, added armbands without restricting shoulder joints, and lowered neckline. Each participant $(n=8)$ was exposed to torso, shoulder, and arm compression, with effects studied through survey and interview questions. As before, the survey ratings showed a trend towards postive valence and calm in terms of arousal. In terms of garment comfort, 6 of 8 participants felt the garment was comfortable and more participants favored actuators that were oriented vertically like backpack straps. Interestingly, an unexpected advantage to mobility with the active garment was provided by the SMAs. When heated, the SMAs contract, pulling the passive fabric close and providing resistance on the body. But when the participant breathes or moves, the actuators (functioning just like springs) extend while still providing compression. Further, since the SMAs operate through heat activation, we also investigated temperature comfort of users. In general, the participants were very receptive to the faint heat of the garment, claiming that they felt calm and relaxed especially when the compression and temperature distribution was consistent. 4 out of 8 of participants even wanted the garment back on after removal (without an explicit question asked), showing that the garment was positively received. Since we are also interested in the ways dynamic compression might be interpreted, we also encouraged free word associations that describe user's feelings when compression was applied to the body as well as potential situations one might expect to use the garment. The most commonly used words include 'comforting', 'cozy', 'calm', 'relaxing', 'warm', 'comfortable', 'exciting', and the sensation was most commonly associated athletic applications, calming therapeutic devices used when stressed, and medical interventions for muscle soreness/pain.

Both studies from Phase 1 demonstrate that our subjects generally welcomed compression on the torso and that the preferences for shoulder regions are dependent on the direction compression is applied. Using SMAs, we found that it was feasible to use a dynamic actuated garment to selectively apply compression on the body. The collected data provides insight into the subjective effects of on-body compression, and the active garment design shows significant potential to be used as an evaluation tool to study compression as a haptic modality. The design implications obtained will be used to inform the future creation and study of a computer-mediated compression garment that is comfortable, wearable, effective, and safe for use. 


\section{EXPECTED CONTRIBUTION IN THE FIELD OF UBIQUITOUS COMPUTING}

The proposed research is expected to contribute to many cross-cutting research areas. Broadly, the design and development of the computer-mediated active compression garment system can be used to inform future designs related to compression systems in the field of wearable computing. From the standpoint of haptic communication and persuasive computing, compression interfaces can potentially take the form of ambient displays, with information presentated in the periphery of human attention. In terms of affective haptics, the generation of new empirical knowledge from the outlined studies will lead to new insights about people's experience with applied computer-mediated on-body compression. Along those lines, this work may also contribute to the field of tele-rehabilitation (i.e., remotely administered compression therapies) and an improved OT treatment paradigm using computer mediated-compression that would not have been possible with traditional, passive compression garments.

\section{REFERENCES}

[1] M. A. Diego, T. Field, C. Sanders, and M. Hernandez-Reif, "Massage Therapy of Moderate and Light Pressure and Vibrator Effects on Eeg and Heart Rate," Int. J. Neurosci, vol. 114, no. 1, pp. 31-44, 2004.

[2] J. R. Hegarty and E. Gale, "Touch as a therapeutic medium for people with challenging behaviours," Br. J. Learn. Disabil., vol. 24, no. 1, pp. 26-32, 1996.

[3] T. Grandin, "Calming effects of deep touch pressure in patients with autistic disorder, college students, and animals.," J. Child Adolesc. Psychopharmacol., vol. 2, no. 1, pp. 63-72, 1992.

[4] T. Field, Touch. Cambridge, MA: MIT Press, 2003.

[5] J. C. Duvall, L. E. Dunne, N. Schleif, and B. Holschuh, "Active 'Hugging' Vest for Deep Touch Pressure Therapy," Proc. 2016 ACM Int. Jt. Conf. Pervasive Ubiquitous Comput. Adjun., pp. 458-463, 2016.

[6] Y. Zheng, E. Su, and J. B. Morrell, "Design and evaluation of pactors for managing attention capture," 2013 World Haptics Conf. WHC 2013, pp. 497-502, 2013.

[7] H. Pohl, P. Brandes, H. N. Quang, and M. Rohs, "Squeezeback: Pneumatic Compression for Notifications," Proc. SIGCHI Conf. Hum. Factors Comput. Syst. - CHI '17, 2017.

[8] M. A. Baumann, K. E. MacLean, T. W. Hazelton, and A. McKay, "Emulating human attention-getting practices with wearable haptics," 2010 IEEE Haptics Symp. HAPTICS 2010, pp. 149-156, 2010.

[9] F. Chinello, M. Aurilio, C. Pacchierotti, D. Prattichizzo, and D. Prattichizzo, "The HapBand: A cutaneous device for remote tactile interaction," Lect. Notes Comput. Sci. (including Subser. Lect. Notes Artif. Intell. Lect. Notes Bioinformatics), vol. 8618, pp. 284-291, 2014.

[10] M. J. Hertenstein, et. al., “Touch communicates distinct emotions.," Emotion, vol. 6, no. 3, pp. 528-533, 2006.

[11] S. Blairs, S. Slater, and D. J. Hare, "The clinical application of deep touch pressure with a man with autism presenting with severe anxiety and challenging behaviour," Br. J. Learn. Disabil., vol. 35, no. 4, pp. 214-220, 2007.

[12] D. Tsetserukou, A. Neviarouskaya, H. Prendinger, N. Kawakami, and S. Tachi, "Affective Haptics in Emotional Communication," Int. Conf. Affect. Comput., no. 1, pp. 181-186, 2009.

[13] G. Huisman, A. Darriba Frederiks, B. Van Dijk, D. Hevlen, and B. Krose, "The TaSSt: Tactile sleeve for social touch," 2013 World Haptics Conf. WHC 2013, pp. 211-216, 2013.

[14] F. "Floyd” Mueller, F. Vetere, M. R. Gibbs, J. Kjeldskov, S. Pedell, and S. Howard, "Hug over a distance," CHI '05 Ext. Abstr. Hum. factors Comput. Syst. - CHI '05, p. 1673, 2005.

[15] J. K. S. Teh, Y. Choi, A. D. Cheok, V. Thuong, R. L. Peiris, and S. Lai, "Huggy Pajama: a mobile parent and child hugging communication system," Proc. 7th Int. Conf. Interact. Des. Child., no. January, pp. 250-257, 2008.

[16] R. Wang and F. Quek, "Touch \& Talk: Contextualizing Remote Touch for Affective Interaction," Proc. fourth Int. Conf. Tangible, Embed. embodied Interact. - TEI '10, pp. 13-20, 2010.

[17] B. J. Fogg, "Persuasive Technologies," Commun. ACM, vol. 42, no. 5, pp. 26-29, 1999.

[18] L. Dunne, "Smart Clothing in Practice: Key Design Barriers to Commercialization," Fash. Pract. J. Des. Creat. Process Fash. Ind., vol. 2, no. 1, pp. 41-66, 2010.

[19] B. Holschuh and D. Newman, "Two-spring model for active compression textiles with integrated NiTi coil actuators," Smart Mater. Struct., vol. 24, no. 3, p. 35011, 2015.

[20] J. H. M. Tulen, P. Moleman, H. G. van Steenis, and F. Boomsma, "Characterization of stress reactions to the Stroop Color Word Test," Pharmacol. Biochem. Behav., vol. 32, no. 1, pp. 9-15, 1989. 


\section{BIOGRAPHICAL SKETCH}

Esther Foo is currently in her second year of doctoral studies in Human Factors and Ergonomics at the University of Minnesota. She started the program in September of 2016 and is expected to complete in May 2020. She obtained her B.Sc. degree in Biomedical Engineering from Purdue University, M.Eng. degree in Biomedical Engineering from Cornell University, and M.Sc. degree in Human Factors and Ergonomics from the University of Minnesota. Esther's research interest lies in the human factors and ergonomics of medical/ healthcare applications and on-body systems. Her specific PhD dissertation research focuses on the objective and subjective effects of dynamic, computer-mediated compression on a user, in the hopes of contributing to cross-cutting research areas including wearable technology, affective haptics, haptic interfaces, and human-computer interaction. Esther is advised by Dr. Brad Holschuh, an Assistant Professor of Wearable Technology and Apparel Design in the College of Design at the University of Minnesota and holds additional graduate faculty appointments in Aerospace Engineering and Mechanics, Human Factors/Ergonomics, and Design. 